\title{
The Drag Reduction by Ultrahigh-Molecular-Weight Polystyrene
}

\author{
Kunio NAKAmURA, Fumitaka YamaShitA, and Tsurutaro NAKAgAwA \\ Department of Polymer Science, Faculty of Science, Hokkaido University, \\ Nishi 8-chome, Kita 10-jo, Kita-ku, Sapporo 060, Japan
}

(Received September 13, 1982)

\begin{abstract}
KEY WORDS Drag Reduction / Ultrahigh-Molecular-Weight Polystyrene / Rolling Ball Apparatus / Temperature Dependence / Turbulent Flow /
\end{abstract}

The reduction of turbulent drag brought about by dissolution of a small amount of a polymer into a fluid is known as drag reduction phenomenon. ${ }^{1}$ Studies on this phenomenon have shown that the Reynolds number at the onset of drag reduction is influenced by flow geometry, ${ }^{2}$ polymer species, polymer concentration, ${ }^{3}$ and polymer molecular weight. $^{4}$

Our previous study ${ }^{5}$ was concerned with the drag reduction of polyelectrolyte solutions measured by a rolling ball viscometer. ${ }^{5}$ The present study was undertaken to investigate drag reduction due to ultrahigh-molecular-weight polystyrene in benzene, with a view to obtaining information on the influence of flow geometry, polymer concentration, and temperature on the Reynolds number at the onset of drag reduction.

\section{EXPERIMENTAL}

Drag reduction was studied using an apparatus consisting of an inclined tube and a rolling ball, which is essentially an instrument for viscosity measurement at high temperatures and pressures. ${ }^{6}$ A general study with this apparatus of laminar and laminar-turbulent flow was reported by Hubburd and Brown. ${ }^{7}$

According to Hubburd and Brown, the drag coefficient $C_{\mathrm{D}}$ and the Reynolds number $R e$ are defined by

$$
\begin{gathered}
C_{\mathrm{D}}=\frac{f}{l^{2} \rho u^{2}} \\
R e=\frac{l u \rho}{\eta}
\end{gathered}
$$

where $l$ is the characteristic length of the flow field and defined by the equivalent diameter usually employed in hydraulics for non-circular channels. This diameter is equal to four times the crosssectional area of the tube divided by the perimeter. The equivalent diameter $l$ of the crescent-shaped space in the present apparatus is defined by

$$
l=4 \frac{\pi\left(D^{2}-d^{2}\right)}{4 \pi(D+d)}=D-d
$$

where $D$ is the tube diameter and $d$, the ball diameter. Since, according to the above-mentioned definition, the characteristic length of a pipe flow is equal to the pipe diameter, the Reynolds number in eq 2 may be compared with the Reynolds number defined in terms of the pipe diameter in the pipe flow. $u$ is the average fluid velocity which is related to the ball velocity $V$ by the equation

$$
\frac{u}{V}=\frac{d^{2}}{D^{2}-d^{2}}
$$

In the above equations, $\rho$ is the density of the fluid, $\eta$, the viscosity of the fluid, and $f$, the force driving the ball represented by

$$
f=\frac{5}{7} \cdot \frac{1}{6} d^{3}\left(\rho_{\mathrm{s}}-\rho\right) g \sin \theta
$$

where the coefficient $5 / 7$ is the fractional gravity that causes the translational motion of the rolling ball, $\rho_{\mathrm{s}}$, the density of the ball, and $\theta$, the angle between the tube and the horizontal plane.

The drag reduction phenomenon was observed in the turbulent flow region by measuring the falling velocity of the rolling ball at various $\theta .{ }^{5}$ The diameter of the glass tube was $1.57 .8 \mathrm{~cm}$ and those of 
the four steel balls used were 1.2, 1.3, 1.4, and $1.5 \mathrm{~cm}$. The value of $7.856 \mathrm{~g} \mathrm{~cm}^{-3}$ at $25^{\circ} \mathrm{C}$ was used for the density of the steel balls.

The polymer sample used in the present investigation was an unfractionated ultrahighmolecular-weight polystyrene, a commercial product IK-1500 of the Japan Synthetic Rubber Co. (JSR). Its molecular weight was $4.8 \times 10^{7}$. The sample was purified by washing with methanol. Benzene was used as the solvent, and the polymer concentration ranged from 11.6 to $93.1 \mathrm{ppm}$ (by weight). The viscosity of the solutions was measured using a Ubbelohde viscometer. No effects of shear degradation on the rheological properties were observed in the present study. For example, the terminal velocity of the $1.2 \mathrm{~cm}$ ball in $93.1 \mathrm{ppm}$ solutions was reproducible within $c a .0 .25 \%$ at $\sin \theta=0.861$.

\section{RESULTS AND DISCUSSION}

The falling velocity of the ball $V$ for simple liquids (benzene, water, water-glycerol) and a 93.1 ppm polymer solution was measured over the range of $\theta$ from $12^{\circ}$ to $75^{\circ}$ at $25^{\circ} \mathrm{C}$, using the balls mentioned above. The drag coefficient $C_{\mathrm{D}}$ and the Reynolds number $R e$ were calculated respectively from eq 1 and eq 2, with the density and viscosity of benzene at $25^{\circ} \mathrm{C}$ taken to be $0.874 \mathrm{~g} \mathrm{~cm}^{-3}$ and 0.602 $\mathrm{mPa}$, respectively. The density of the solution assumed to be that of benzene, and the relative viscosity is shown in Table I.

The variation of $\log C_{\mathrm{D}}$ with $\log R e$ is shown in Figure 1. The values of $\log C_{\mathrm{D}}$ for benzene decrease with increasing $R e$ and show breaks at high Reynolds numbers for the $1.2,1.3$, and $1.4 \mathrm{~cm}$ balls (see dotted lines). This behavior was also noted in the $\log C_{\mathrm{D}} v s . \log R e$ plot for the $93.1 \mathrm{ppm}$ solution. The value of $R e$ at the break point for benzene with the $1.2 \mathrm{~cm}$ ball corresponds to a $\theta$ of $c a .70^{\circ}$. Above this angle, the motion of the ball became visibly irregular and the slip of the ball was observed.

\section{Effect of Equivalent Diameter on Drag Reduction}

By dimensional analysis, the following relationship was obtained by Hubburd and Brown ${ }^{7}$ :

$$
\frac{f}{l^{2} \rho u^{2}}=F\left(\frac{l u \rho}{\eta}\right)
$$

The form of the function $F$ is complicated, but may
Table I. Reynolds numbers at the onset of drag reduction for IK-1500 solutions in benzene; the equivalent diameter of the tube was $0.378 \mathrm{~cm}$

\begin{tabular}{ccccc}
\hline Temperature & Concentration & $\begin{array}{c}\text { Relative } \\
\text { viscosity }\end{array}$ & $\begin{array}{c}\text { Reynolds } \\
\text { number }\end{array}$ \\
\cline { 1 - 2 }${ }^{\circ} \mathrm{C}$ & $\mathrm{ppm}$ & & 1.008 & 1900 \\
25 & 11.6 & 1.024 & 1680 \\
25 & 23.3 & 1.077 & 1470 \\
25 & 46.6 & 1.151 & 1120 \\
25 & 93.1 & 1.075 & 1920 \\
45 & 46.6 & 1.098 & 1120 \\
6 & 46.6 & & \\
\hline
\end{tabular}

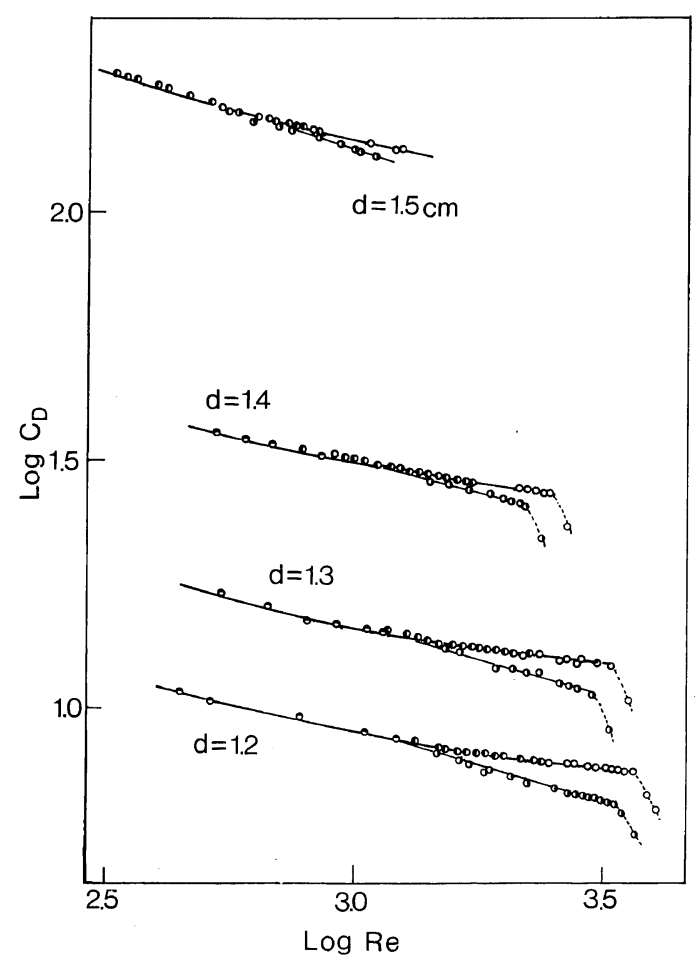

Figure 1. $\log C_{\mathrm{D}}$ plotted against $\log R e$ for simple fluids and $93.1 \mathrm{ppm}$ solutions of IK-1500 in benzene, measured with steel balls of various sizes at $25^{\circ} \mathrm{C}$ : $O$, benzene; $\boldsymbol{D}$, water; $\Theta$, water-glycerol; $\bigcirc, 93.1 \mathrm{ppm}$. $D$ is $1.57_{8} \mathrm{~cm}$.

practically be represented by an experimental $\log C_{\mathrm{D}}$ vs. $\log R e$ plot. Hubburd and Brown showed that this plot in the laminar region gives a straight line of slope -1 and that in the turbulent region follows a smooth concave curve. The $\log C_{\mathrm{D}}$ vs. $\log R e$ data 
for simple liquids (benzene, water, water-glycerol) showed features characteristic of the turbulent flow, as can be seen from Figure 1. Moreover, these data could be reduced to a single curve. On the other hand, the data for the $93.1 \mathrm{ppm}$ solution for various ball diameters fell below the lines for simple Newtonian fluids. This is a clear demonstration of drag reduction. The Reynolds number at the onset of drag reduction was obtained from the intersection of the two straight lines fitting the data points for the simple liquids and the polymer solution. Figure 1 shows that $R e$ at the onset of drag reduction decreases as the ball diameter increases or as the equivalent diameter defined by eq 3 decreases. Similar results were obtained for pipe flows of other polymers. $^{2,8}$

\section{Concentration Effects on Drag Reduction}

Figure 2 shows the $R e$ dependence of the drag coefficient obtained with the $1.2 \mathrm{~cm}$ ball for polymer

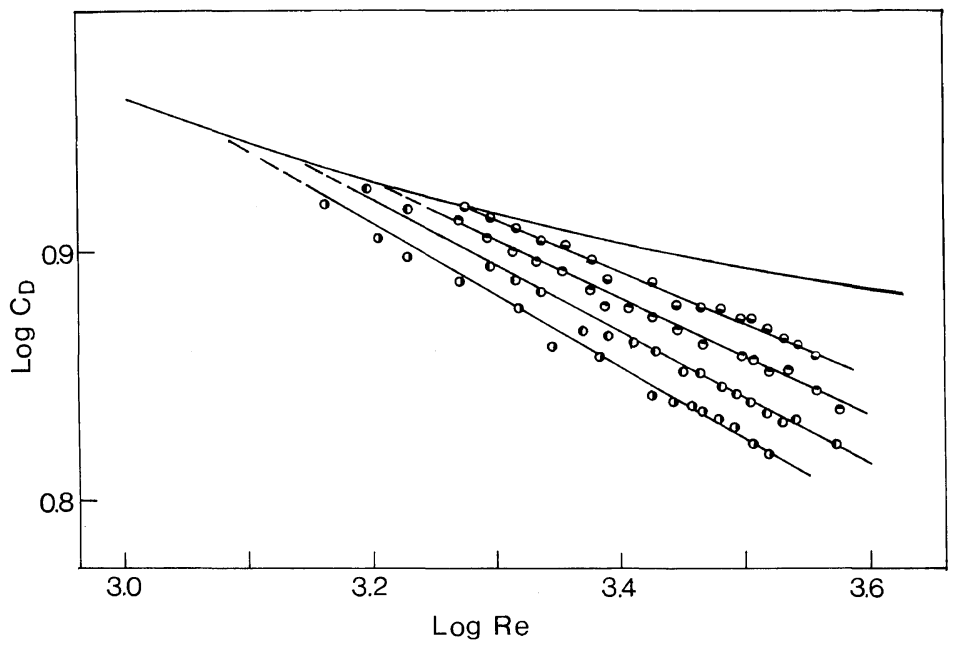

Figure 2. $\log C_{\mathrm{D}}$ plotted against $\log R e$ for various concentrations of $\mathrm{IK}-1500$ in benzene at $25^{\circ} \mathrm{C} . D$ and $d$ are $1.57_{8}$ and $1.2 \mathrm{~cm}$, respectively; $\bigcirc$, benzene; $\ominus, 11.6 ; \ominus, 23.3 ; \boldsymbol{O}, 46.6 ; \bigcirc, 93.1 \mathrm{ppm}$.

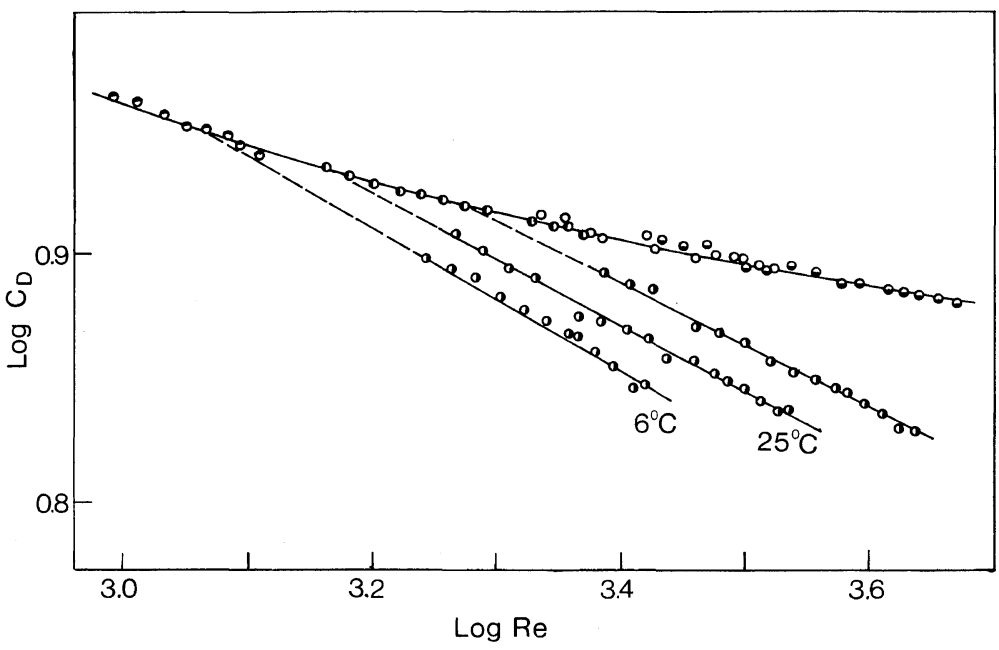

Figure 3. $\log C_{\mathrm{D}}$ plotted against $\log R e$ for simple fluids and $46.6 \mathrm{ppm}$ solutions of IK-1500 in benzene at various temperatures: $\ominus$, benzene at $45^{\circ} \mathrm{C}$; $\bigcirc$, benzene; $\odot$, water; $\Theta$, water-glycerol at $25^{\circ} \mathrm{C} ; \boldsymbol{O}, 46.6$ ppm. $D$ and $d$ are $1.57_{8}$ and $1.2 \mathrm{~cm}$, respectively. 
solutions and simple liquids. It can be seen that the values of $\log C_{\mathbf{D}}$ - decrease linearly with increasing $\log R e$ and that the absolute value of the slope increases as the polymer concentration increases. The $R e$ at the onset of drag reduction is listed in Table I. This critical $R e$ decreases with increasing polymer concentration, in conformity with the currently available experimental data for the pipe flow. ${ }^{3}$

\section{Temperature Effect on Drag Reduction}

Figure 3 illustrates the temperature effect on drag reduction. The $C_{\mathrm{D}} v s$. Re data for benzene at various temperatures fall on a single curve. On the other hand, data for the $46.6 \mathrm{ppm}$ solution show temperature-dependent behavior. The Reynolds number at the onset of drag reduction as a function of temperatures is given in Table $\mathrm{I}$.

In summary, the present study shows that drag reduction occurs at a lower $R e$ as the equivalent diameter decreases, the polymer concentration increases, and the temperature decreases, in the case of ultrahigh-molecular-weight polystyrene in benzene. In particular, the $R e$ at the onset of drag reduction was found to be a function not only of the equivalent diameter and the polymer concentration but also of temperature. Thus, the dimension and deformability of polymer coils in solution may be responsible for the onset of drag reduction.

Acknowledgement. The authors wish to thank Mr. K. Arai of the Japan Synthetic Rubber Co. for supplying the samples.

\section{REFERENCES}

1. B. A. Toms, "Proceedings of the 1st International Congress on Rheology, Vol. II, North Holland, Amsterdam, 1949, p 135.

2. R. S. Sharma, V. Seshadri, and R. C. Malhatra, Polymer, 20, 257 (1979).

3. R. C. Little, R. J. Hansen, D. L. Hungton, O-K. Kim, R. L. Patterson, and R. Y. Ting, Ind. Eng. Chem. Fundam., 14, 283 (1975).

4. P. S. Virk and E. W. Merrill, "Viscous Drag Reduction," C. S. Wells, Ed., Plenum Press, New York, 1969, p 107.

5. F. Yamashita, K. Nakamura, and T. Nakagawa, Rep. Prog. Polym. Phys. Jpn., XXIV, 93 (1981).

6. K. Kubota and K. Ogino, Macromolecules, 12, 74 (1979).

7. R. M. Hubburd and G. G. Brown, Ind. Eng. Chem., Anal. Ed., 15, 212 (1943).

8. H. C. Hershey and J. L. Zakin, Chem. Eng. Sci., 22, 1847 (1967). 